\title{
Is Digital Subtraction Flouroscopy a Useful Tool for the Interventional Pain Physician?
}

Joseph F. Jasper, MD

Most interventional pain physicians are experts in the use of fluoroscopy and understand its importance in improving the safety, accuracy and efficacy of diagnostic and therapeutic procedures. The addition of digital subtraction may enhance the utility of radiography and improve the accuracy of our interpretations. This article illustrates the use of

Fluoroscopy has become the standard for administering diagnostic and therapeutic spinal injections. The strength of evidence for the efficacy of various modes of spinal injection supports this, as detailed by Manchikanti et $\mathrm{al}^{(1)}$. Typically, the use of fluoroscopy is coupled to the injection of some radiological contrast to document proper placement.

In the case of epidural steroid injections by all routes this should result in an epidurogram. The epidurogram confirms proper placement but may also be useful in outlining filling defects that may assist diagnostically.

Digital pictures can be enhanced with a variety of controls such as contrast and brightness. Also arithmetic summations, differences or subtractions may combine two or more images of the same region pre- and postcontrast injection to yield an improved analysis of contrast spread. This is most commonly applied in angiography as recently reviewed by Gates and Hartnell (2). There may be a further role to explore in terms of the use of carbon dioxide as a digital subtraction contrast for patients allergic to injectable contrast agents, as described by Barbey et al (3); perhaps this could be even more safely applied in the epidural space than via intravascular method. The problem of motion artifact is reviewed by Meijering et al (4).

The claimed benefit of caudal adhesiolysis technique is

From Advanced Pain Medicine Physicians, Tacoma, Washington. Dr. Jasper is the medical director at Advanced Pain Medicine Physicians. Address Correspondence: Joseph Jasper, MD, 1628 South Mildred Street \#105, Tacoma, WA 98465. E-mail: apmedicine@ qwest.net the technique in several patients. The utility of this enhanced technique in clinical versus research application remains to be determined.

Keywords: Interventional pain medicine, fluoroscopy, epidural steroids, epidurogram

that areas of filling defect on the initial epidurogram are overcome by catheter placement, manipulation and hydrostatic forces and, possibly, chemo-adhesiolysis (5); though evidence for this last point is weak. Digital subtraction can highlight the distribution of an epidurogram clearly.

\section{ILLUSTRATION OF DIGITAL SUBTRACTION}

The images accompanying this article were obtained before and after contrast injection and were downloaded to floppy disk. Digital subtraction was achieved with imaging software on a computer. Some fluoroscopy units have optional digital subtraction packages that would likely perform these steps automatically and faster.

Digital subtraction may be utilized in multiple areas in interventional pain medicine. Potential applications are as follows:

- Epidural injections,

- Discography, and

- Adhesiolysis.

Three illustrations using digital substraction of caudal epidural steroid injections with the catheter tip in the anterior epidural space near the left L5 pedicle are shown in Fig. 1.

Subtraction of the preinjection image from the postinjection image eliminates the majority of bone shadows. The filling defect at left L5-S1 is far more apparent on the subtraction image than on the plain epidurogram. To achieve this, the patient and fluoroscopy tube must be immobile and the images obtained at the same respiratory stage. Movement degrades the utility of this technique. 


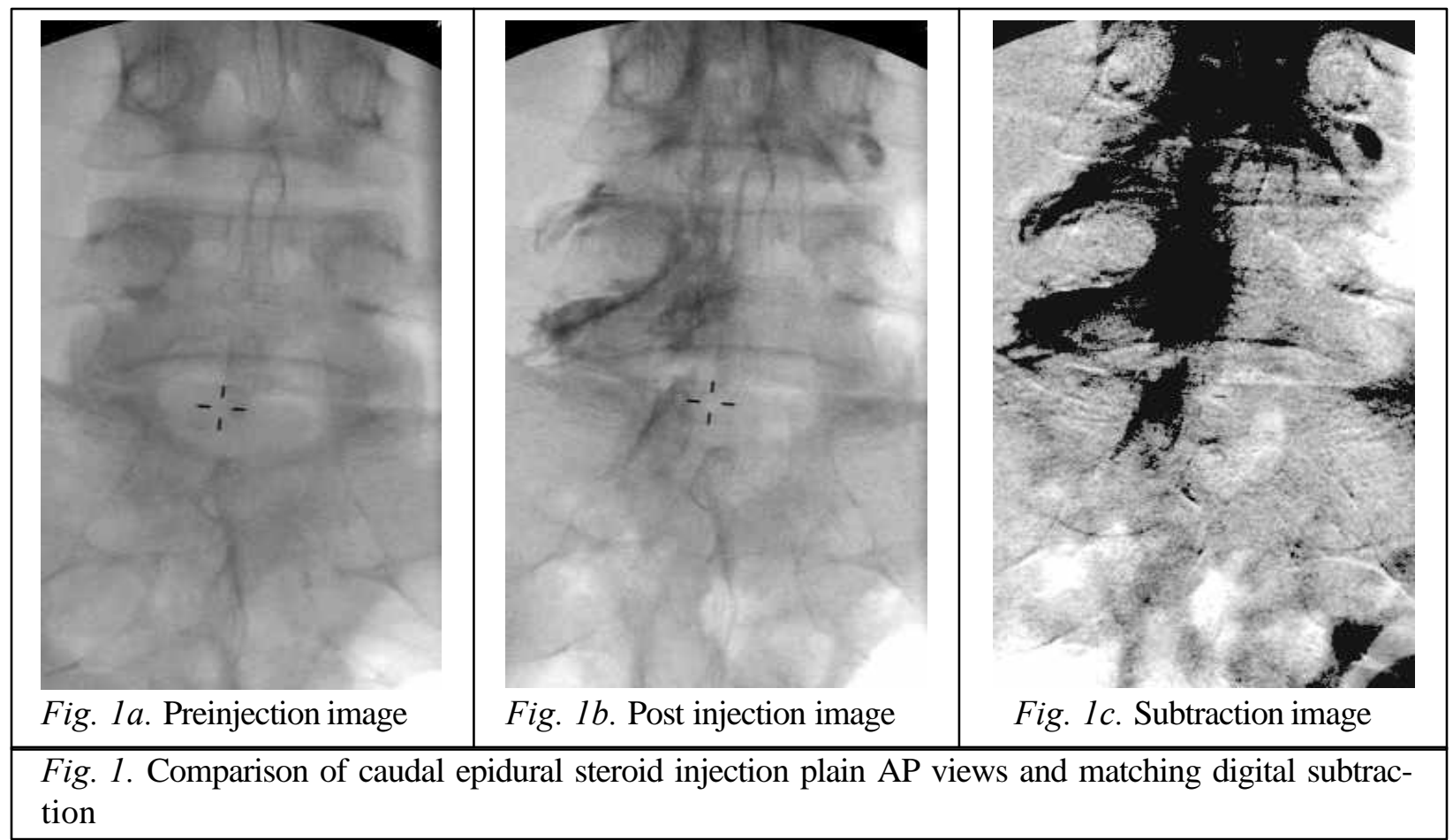

Lateral subtraction views are also useful. The L5-S1 anterior epidural region is often difficult to see due to the traversing ilium and large articular processes. As seen in Fig. 2 the filling defect is clearly defined with subtraction. In this patient a large L5-S1 disc herniation is present on magnetic resonance imaging (MRI), (Fig. 2 a-b).

A filling defect such as this implies only a resistance to flow, with contrast following the path of least resistance. The contrast defect could be partially disc, partially ligament, induration, scar tissue or tumor.

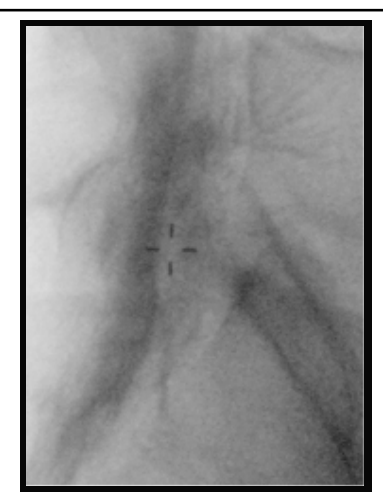

Fig. 2a. MRI image
Fig. 2b. Subtraction Image

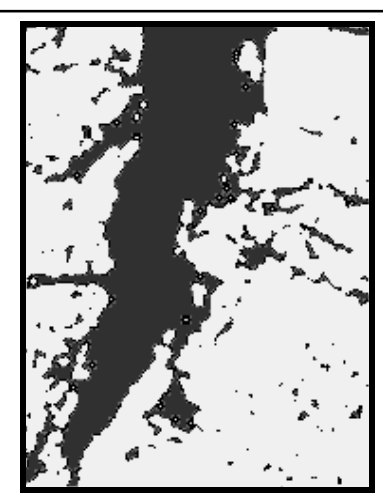

Fig. 2. Comparison of lateral views
Fig. 3 "a-d" shows plain anteroposterior views during the course of a caudal adhesiolysis, while "e-g" show digital subtractions. Fig. 3a is preinjection, and $3 \mathrm{~b}$ follows $10 \mathrm{cc}$ of caudal injected contrast before catheter placement. Fig. $3 e$ is the subtraction of 3b-3a. Fig. $3 c$ follows catheter introduction and injection of several $\mathrm{cc}$ of contrast into the filling defect and area of greatest symptoms at left L5. Fig. $3 \mathrm{f}$ is the subtraction of $3 \mathrm{c}-3 \mathrm{a}$, revealing total contrast spread with the precatheter and postadhesiolysis injections. Fig. $3 \mathrm{~g}$ is the result of subtraction of $3 \mathrm{c}-3 \mathrm{~b}$, demonstrating only the new areas filled following adhesiolysis. Fig. 3d follows $15 \mathrm{cc}$ additional contrast and saline adhesiolysis. No release of the central L5-S1 adhesion occurred with this additional volume.

Cervical epidural steroid injections studied in this way are quite revealing. A typical AP epidural pattern perceived with conventional fluoroscopy for interlaminar epidural steroid injection with catheter typically reveals a "ladder"like appearance and insignificant central or anterior spread. With subtraction far more diffuse spread is appreciated, and one better appreciates epineural sleeve filling. On regular lateral films, anterior spread is hard to appreciate, as the shoulders and head add a great deal of overlapping shadows. Subtraction reveals considerably greater central and epineural spread than would otherwise be appreciated (Fig. 4a-c). 


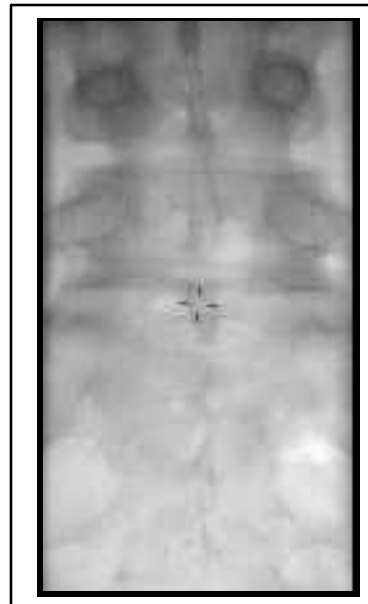

Fig. 3a. Preinjection

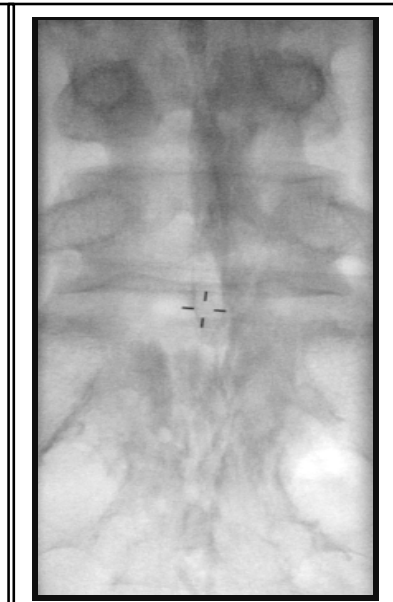

Fig. $3 b$. Following $10 \mathrm{~mL}$ of contrast prior to catheter

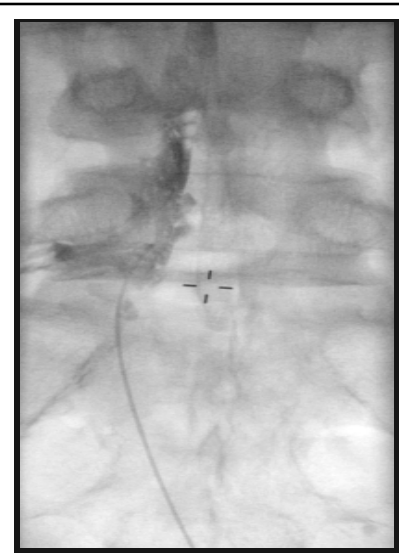

Fig. 3c. Following catheter introduction and injection of several $\mathrm{mL}$ of contrast into filling defect

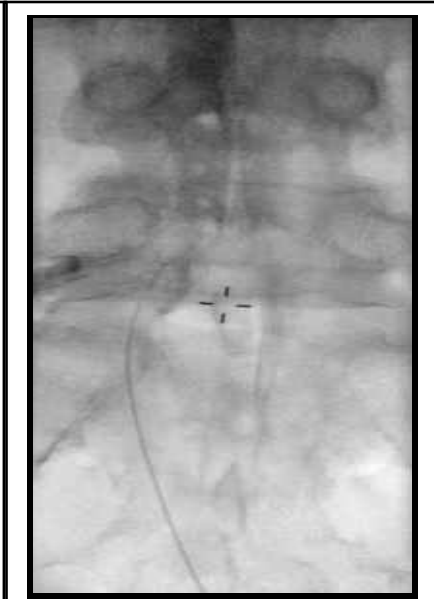

Fig. 3d. Following additional $15 \mathrm{~mL}$ contrast and saline adhesiolysis

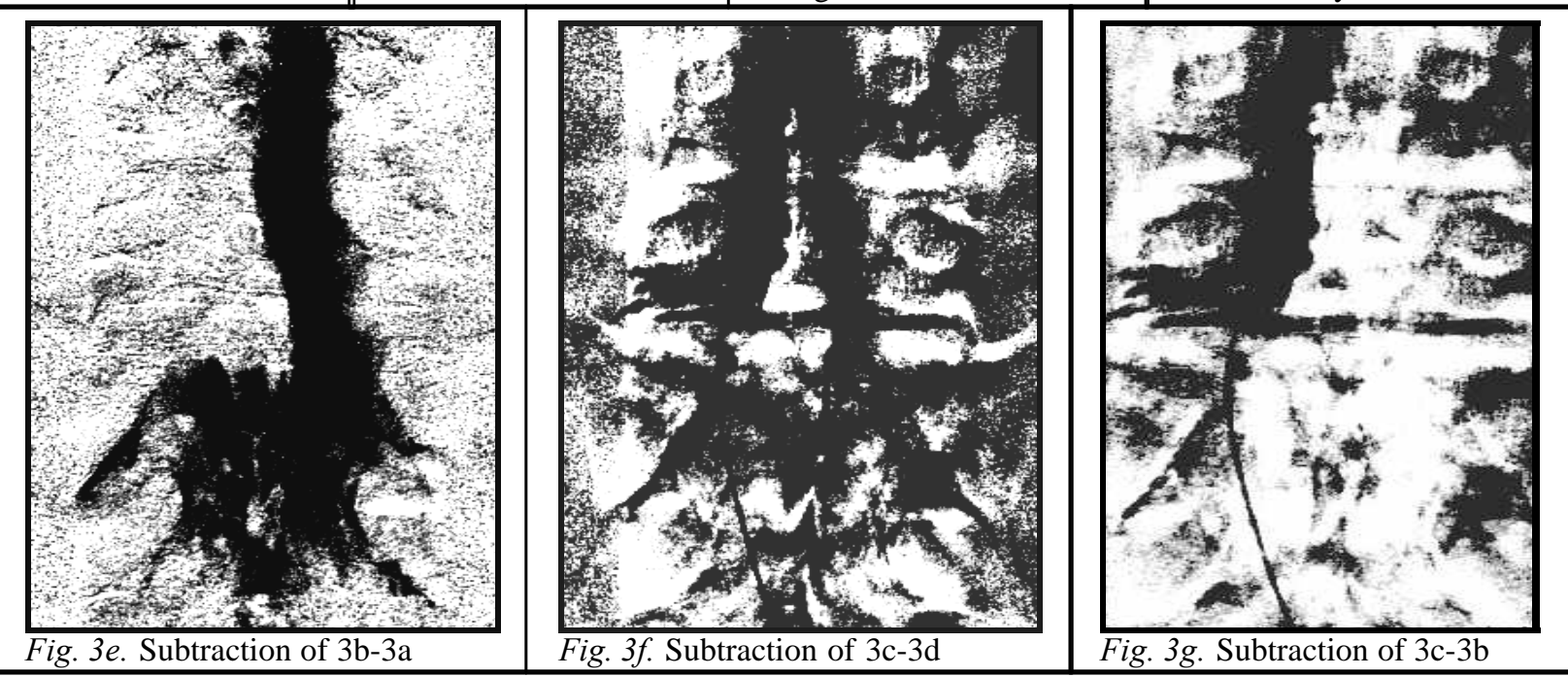

Fig. 3. Comparison of plain AP views during caudal adhesiolysis and matching digital subtractions

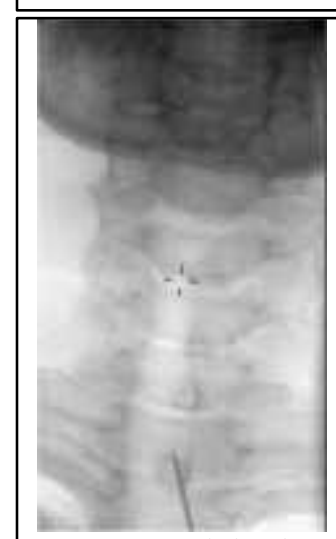

Fig. 4a. Preinjection

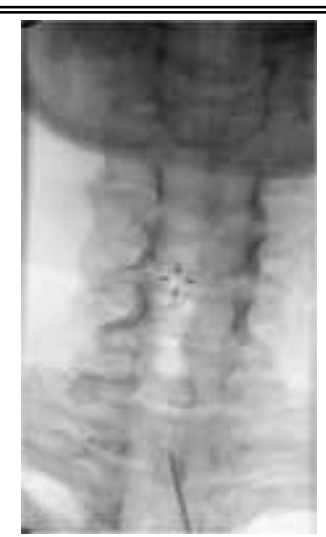

Fig. 4b. Post injection

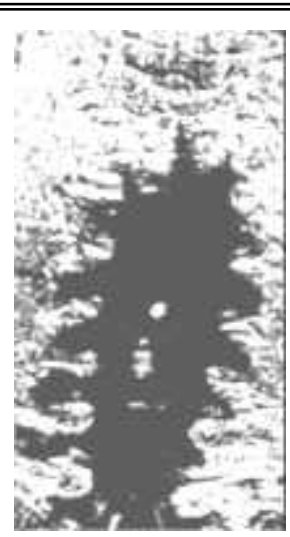

Fig. 4c. Subtraction

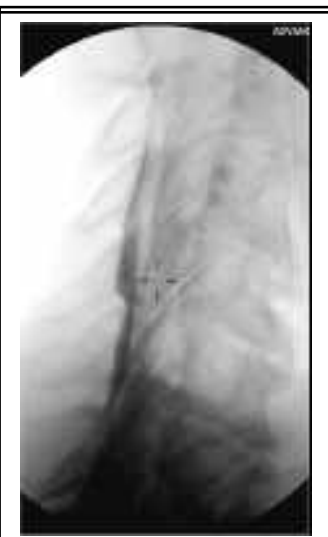

Fig. 4d. Regular lateral film

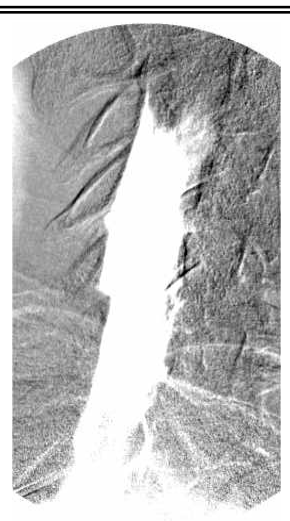

Fig. 4e. Subtraction

Fig. 4. Comparison of AP and lateral views during cervical epidural steroid injection and matching digital subtractions 


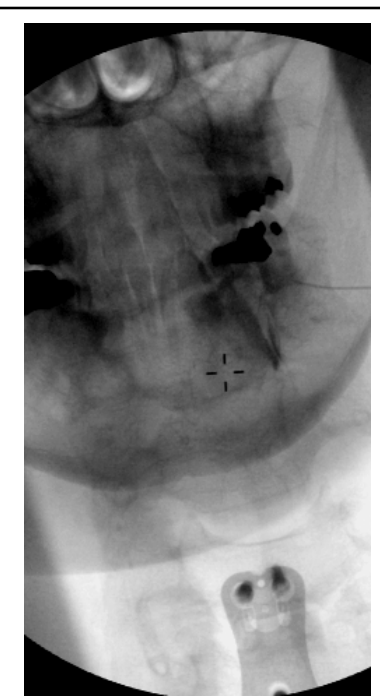

Fig. 5a. Transforaminal injection, regular film

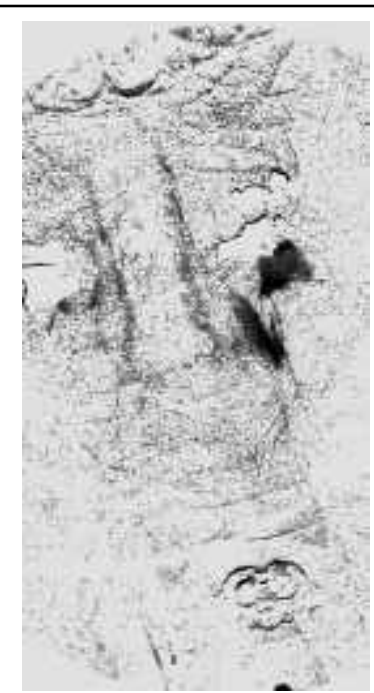

Fig. 5b. Transforaminal injection, subtraction

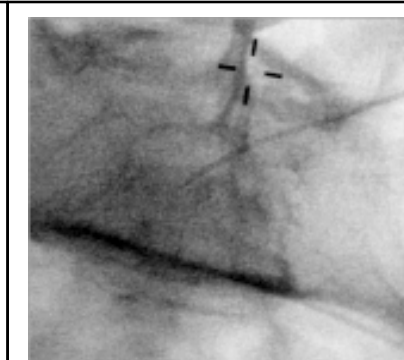

Fig 6a. Preinjection

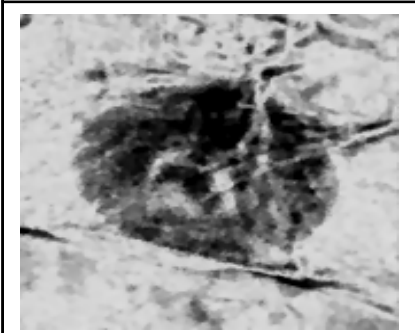

Fig 6c. Subtraction

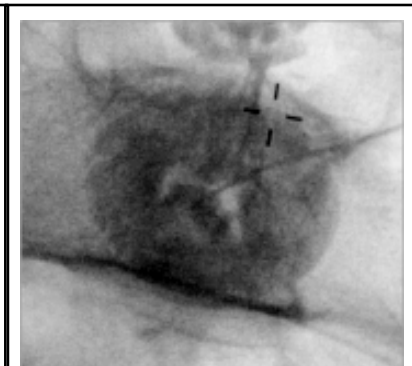

Fig 6b. Post injection

Fig. 6. The pre and post injection images and a digital subtraction for one patient with demonstration of an annular tear already suspected by MRI
Subtraction is also helpful on a transforamenal injection, particularly at C3, as demonstrated in Fig. 5a-b.

Discography, a diagnostic and surgical planning tool, is primarily performed to demonstrate provocation of concordant pain via pressurization of the lumbar discs. It is considered a prerequisite test to fusion, annuloplasty or nucleoplasty. In addition to documenting the level of pain generation, it can demonstrate morphologic characteristics (6). This can be on plain films, or with the controversial and expensive addition of computerized tomography scanning postdiscography. Obtaining axial-like views preand postintradiscal contrast injection, one can subtract out the distracting elements and demonstrated pathology. With this technique the same requirement exists for maintaining the fluoroscopy and patient positions and respiratory cycle timing. Because some patients move when stimulated by provocative discography, this technique may have limitations.

Fig. 6 in left to right sequence are the pre- and postinjection images and a digital subtraction for one patient with demonstration of an annular tear already suspected from MRI.

\section{CONCLUSION}

Most interventional pain physicians are comfortable with fluoroscopy use and understand its importance in improving the safety, accuracy and efficacy of diagnostic and therapeutic procedures. The addition of digital subtrac- tion may enhance the utility of radiography and the accuracy of our interpretations. There exists the possibility of using carbon dioxide as a contrast agent in patients sensitive to even nonionic contrast media, but this remains to be explored in interventional pain applications. Digital subtraction fluoroscopic interventional pain procedures could result in improved patient diagnosis, therapy assignment and therapeutic outcome. Further studies need to explore the actual clinical utility of digital subtraction for interventional pain procedures.

\section{REFERENCES}

1. Manchikanti L, Singh V, Kloth Det al. Interventional Techniques in the Management of Chronic Pain: Part 2.0. Pain Physician 2001;4:24-96.

2. Gates J, Hartnell GG. Optimized diagnostic angiography in high risk patients with severe peripheral vascular disease. Radiographics 2000;20:121-33.

3. Barbey MM, Farber A, Marienhoff N et al. [Digital subtraction angiography with carbon dioxide-Basic principles, technique and clinical application ( in German) Vasa . 1999;28:243-249.

4. Meijering EH, Niessen WJ, Viergever MA et al. Retrospective motion correction in digital subtraction angiography: review. IEE Trans Med Imaging 1999;18:221.

5. Anderson SR, Racz GB, Heavner J. Evolution of epidural lysis of adhesions. Pain Physician 2000; 3:262270.

6. Fortin JD. Precision diagnostic disc injections. Pain Physician 2000; 3:271-288. 